\title{
On the elicitation of indirect preferences in interactive evolutionary multiple objective optimization e-Appendix (supplementary material)
}

\author{
Michał K. Tomczyk, Miłosz Kadziński \\ Institute of Computing Science, Poznań University of Technology \\ Poznań, Poland \\ \{michal.tomczyk,milosz.kadzinski\}@cs.put.poznan.pl
}

ACM Reference Format:

Michał K. Tomczyk, Miłosz Kadziński. 2020. On the elicitation of indirect preferences in interactive evolutionary multiple objective optimization e-Appendix (supplementary material) . In Genetic and Evolutionary Computation Conference (GECCO '20), fuly 8-12, 2020, Cancún, Mexico. ACM, New York, NY, USA, 3 pages. https://doi.org/10.1145/3377930.3389826

In the e-Appendix, we discuss the material which - for the sake of conciseness - was not included in the main paper. Specifically, we:

(1) discuss computational complexities of the proposed procedures enhancing IEMO/D (Section 1),

(2) provide implementation details of the $G P-I G^{t h}$ questioning strategy (Section 2),

(3) compare the results attained by IEMO/D coupled with $I G^{t h}$ and $G P-I G^{\text {th }}$ (Section 3),

(4) explain how we generated the preference intensity thresholds used during the elicitation step to compare differences between scores attained by the reference solutions (Section 4).

\section{COMPUTATIONAL COMPLEXITIES OF THE PROPOSED PROCEDURES}

In this section, we discuss the computational complexities of the proposed procedures enhancing the original IEMO/D algorithm. Firstly, let us remind the computational complexity of IEMO/D. This algorithm consists mainly of two parts: an incorporated evolutionary framework and a Monte Carlo procedure for generating new optimization goals [2]. Their average computational complexities are as follows:

- evolutionary framework: IEMO/D is based on MOEA/D. Hence, its computational complexity at one generation is $O(M T N)$, where $M$ is the number of objectives, $T$ is the neighborhood size, and $N$ is the population size.

- procedure for generating new optimization goals: Every time the Decision Maker (DM) provides a new preference example, the set of optimization goals maintained by

Permission to make digital or hard copies of all or part of this work for personal or classroom use is granted without fee provided that copies are not made or distributed for profit or commercial advantage and that copies bear this notice and the full citation on the first page. Copyrights for components of this work owned by others than ACM must be honored. Abstracting with credit is permitted. To copy otherwise, or republish, to post on servers or to redistribute to lists, requires prior specific permission and/or a fee. Request permissions from permissions@acm.org.

GECCO '20, July 8-12, 2020, Cancún, Mexico

(C) 2020 Association for Computing Machinery.

ACM ISBN 978-1-4503-7128-5/20/07 . \$ \$15.00

https://doi.org/10.1145/3377930.3389826
IEMO/D has to be revised. If some optimization goals do not remain compatible, IEMO/D uses a rejection sampling technique based on Monte Carlo simulation with the aim of generating compatible goals in their place. It is difficult to provide an average computational complexity for this process. The reason is that the success rate of the rejection sampling procedure depends on various factors, e.g., the number of objectives $M$, the number of pairwise comparisons in $\mathcal{H}$, the similarity of solutions compared by the DM, and the upper limit LIM for the number of random samples this procedure is allowed to generate. Hence, we discuss its pessimistic computational complexity. It corresponds to a situation when the sampling procedure reaches the upper limit LIM. This happens when the first $L I M-|\mathcal{H}|$ samples prove incompatible, while the last $|\mathcal{H}|$ samples prove compatible. Both situations require, respectively, $2 M(L I M-|\mathcal{H}|)(|\mathcal{H}|-1)=$ $2 M\left(L I M \cdot|\mathcal{H}|-L I M-|\mathcal{H}|^{2}+|\mathcal{H}|\right)$ and $2 M\left|\mathcal{H}^{2}\right|$ computational steps. Consequently, the pessimistic complexity of the procedure for generating new compatible model instances is $O(M \cdot L I M \cdot|\mathcal{H}|)$.

In what follows, we provide computational complexities of the proposed procedures enhancing IEMO/D.

Procedures for selecting reference solutions to be critically judged by the DM.

- Medoid Selection (MS): $M S$ tests all $N(N-1) / 2$ pairs of solutions to find the one that is the most suitable as a representative of two different regions in the objective space. Having a pair of candidate solutions representing two clusters, MS assigns the remaining solutions to one of these clusters. This requires $2 M(N-2)$ steps. Hence, the computational complexity of the $M S$ procedure is $O\left(M N^{3}\right)$.

- Pairwise Winning Index based on Targets (PWIT): As with $M S, P W I T$ iterates over all pairs of solutions, i.e., $N(N-$ $1) / 2$, and selects the one for which the uncertainty on which solution is preferred is the greatest. Specifically, having a pair of solutions, PWIT compares them using all optimization goals in $\mathcal{L}$. This requires $2 M N$ comparisons. Consequently, the overall computational complexity of PWIT is also $O\left(M N^{3}\right)$.

Deciding when the DM should be questioned for preference information.

- Improved Goals (IG): $I G$ decides upon the questioning of the DM based on the share of optimization goals that have been successfully improved in the current generation. Since 
it does not require any an additional computations because the change in optimization goals can be traced already during the solution replacement step performed by IEMO/D, the computational complexity of $I G$ is $O(1)$.

- Improved Goals combined with Geometric Progression (GP-IG): As with $I G$, the computational complexity of $G P$ $I G$ is $O(1)$. However, in contrast to $I G, G P-I G$ analyses the updates of maintained optimization goals to trigger only the first interaction. The remaining interactions are triggered using a static, pre-defined interaction pattern and, thus, the computational complexity remains $O(1)$.

\section{THE IMPLEMENTATION DETAILS OF THE $G P-I G^{t h}$ QUESTIONING STRATEGY}

The $G D-I G^{t h}$ questioning strategy distributes the interactions with the DM according to the following two rules:

(1) The first interaction is triggered when the progress of the evolutionary search is not sufficient, i.e., when the $\delta$ indicator is lesser than the assumed threshold $t h$.

(2) Having triggered the first interaction, $G D-I G^{t h}$ distributes the remaining ones so that the subsequent elicitation intervals form a geometric progression.

On the one hand, the geometric progression allows constructing elicitation intervals of a similar size, which is desired [1]. On the other hand, depending on when the first interaction is performed, the method may suitably shift the interactions toward the early or late stages of the optimization process, adapting in this way to the difficulty of the underlying Multiple Objective Optimization (MOO) problem. In what follows, we explain how $G D-I G^{t h}$ decides when the DM should be questioned for preference information.

Let us denote by $G$ and $L$, respectively, the number of generations IEMO/D is run for and the maximal allowed number of interactions with the DM. We define a vector $I=\left[0, g_{1}, g_{2}, \ldots, g_{L}, G\right]$, where $g_{j}$ indicates in which generation, the $j^{\text {th }}$ interaction with the DM should be triggered $\left(I_{j}>I_{j-1}\right.$ for all $\left.j=2, \ldots, L+1\right)$. Further, let $\tau_{j}$ denote an elicitation interval between $(j)^{t h}$ and $(j+1)^{t h}$ interactions, i.e., $\tau_{j}=I_{j+1}-I_{j}$. Having triggered the first interaction, $G D-I G^{t h}$ instantiates $g_{1}$ and thus $\tau_{1}$. Then, it builds a geometric sequence of elicitation intervals in the following way:

$$
\begin{array}{r}
\forall_{j \in\{2, \ldots, L+1\}} \tau_{j} / \tau_{j-1}=q, \\
\text { s.t. } q>0 \text { and } \sum_{j=1, \ldots, L+1} \tau_{j}=G .
\end{array}
$$

The value of $q$ is unknown and must be calculated. Let $g(q)=$ $\sum_{j=1, \ldots, L+1} \tau_{j}=\tau_{1}+\tau_{1} q+\tau_{1} q^{2}, \ldots, \tau_{1} q^{L}$. Then, let us define an error function: $E(q)=g(q)-G$. Since $g^{\prime}(q)$ is monotonic, $|E(q)|$ has just a single optimum. For this reason, any greedy optimization algorithm should guarantee finding an optimal solution. In this paper, we simply initialize $q$ with 1 and iteratively increase or decrease it by some $\Delta q$ value until $E(q)$ is sufficiently small. If $E(1)>0$, then we initialize $\Delta q$ with -0.5 as $q$ should be decreased. Otherwise, $\Delta q$ is initialized with 0.5 since $q$ has to be increased. Then, we iteratively verify if $E(q+\Delta q) E(q)<1$. If so, then the procedure has added (subtracted) too much to (from) $q$. In this case, we divide $\Delta q$ by two. If the condition is not satisfied, we update $q$ in the following way: $q=q+\Delta q$. The process is repeated until $E(q)$ is lesser than $10^{6}$, which is considered a satisfactory error level. When a fine approximation of $q$ is found, $G D-I G^{t h}$ computes the elicitation intervals used to decide when the DM should be questioned for preference information.

\section{COMPARISON OF IEMO/D COUPLED WITH $I G^{t h}$ AND $G P-I G^{t h}$}

In this section, we compare the performance of IEMO/D coupled with $I G^{t h}$ and $G P-I G^{t h}$. As in the main paper, we consider the following values for the threshold: $t h \in\{1 \%, 5 \%, 10 \%, 20 \%, 30 \%\}$. In Figure 1, we illustrate the average number of interactions performed by both algorithms when applied to WFG3 for $M=3$. Note that we also depict the results for IEMO/D when coupled with the benchmark RI interaction policy. Let us start by discussing the results for IEMO/D coupled with $I G^{\text {th }}$ (see Figure 1a). This algorithm does not guarantee that the expected number of interactions is performed. As a consequence, the performance of IEMO/D is deteriorated. This may happen when the threshold $t h$ is relatively low. For instance, IEMO/D coupled with $I G^{1 \%}$ triggered only around 6 interactions. In turn, when th is too high, the interactions are performed one after another (see, e.g., the results for $I G^{20 \%}$ and $I G^{30 \%}$ in Figure 1a). Consequently, IEMO/D does not have enough time to make proper use of the most recent DM's answer, which negatively affects its performance. Let us now focus on the results for IEMO/D coupled with $G P-I G^{t h}$ (see Figure $1 \mathrm{~b}$ ). Unlike $I G^{t h}$, $G P-I G^{t h}$ guarantees that the required number of interactions is performed. Moreover, these interactions are sparsely distributed throughout the evolutionary process. Nevertheless, depending on when the first interaction is triggered - which is strictly related to the underlying problem difficulty - the method may suitably shift the interactions toward early (see the results for $G P-I G^{10 \%}$ and $G P-I G^{20 \%}$ in Figure 1b) or late (see the results for $G P-I G^{1 \%}$ in Figure $1 \mathrm{~b}$ ) stages of the optimization process.

In Tables 1 and 2, we provide results of a comprehensive experimental evaluation involving 100 runs of the algorithms for each considered scenario. The methods were applied to WFG1 (Table 1) and WFG3 (Table 2) benchmarks with $M=2, \ldots, 5$ objectives. In this experiment, we directly compared $I G^{t h}$ with $G P$ $I G^{t h}$ parameterized with the same thresholds $t h$ to reliably assess which algorithm performs better. The results indicate that IEMO/D coupled with $G P-I G^{\text {th }}$ performs significantly better than when coupled with $I G^{t h}$. Specifically, the former algorithm's variant outperformed the latter for the vast majority of considered scenarios and proved to be no worse for the remaining ones. The exceptions in this regard are three scenarios involving WFG3 with $M=3$ and th $\in\{5 \%, 10 \%, 20 \%\}$, where IEMO/D coupled with $I G^{t h}$ slightly performed more advantageous than the remaining algorithm (see Table 2).

\section{GENERATING THRESHOLDS FOR PREFERENCE INTENSITY STATEMENTS}

During the experimental verification, we used an $L_{\infty}^{w^{D M}}$-norm incorporating a pre-generated weight vector $w^{D M}$ to simulate the DM's 
Table 1: Average BRSD and ARSD and ranks $\bar{R}$ attained by IEMO/D coupled with $I G^{t h}$ and $G D-I G^{\text {th }}$ parameterized with different thresholds, when applied to WFG1 problem involving from 2 to 5 objectives.

(a) BRSD

\begin{tabular}{|c|ccc|ccc|ccc|ccc|c|}
\cline { 2 - 15 } \multicolumn{1}{c|}{} & \multicolumn{4}{|c|}{$M=2$} & \multicolumn{3}{c|}{$M=3$} & \multicolumn{3}{c|}{$M=4$} & \multicolumn{3}{c|}{$M=5$} \\
\hline Procedure & Mean & StD & $\bar{R}$ & Mean & StD & $\bar{R}$ & Mean & StD & $\bar{R}$ & Mean & StD & $\bar{R}$ \\
\hline$I G(1 \%)$ & 18.92 & 9.94 & 1.41 & 10.14 & 6.40 & 1.18 & 6.73 & 4.37 & 1.07 & 5.59 & 3.64 & 1.21 \\
$G P-I G(1 \%)$ & 19.17 & 8.89 & 1.59 & 12.95 & 8.13 & 1.82 & 10.47 & 6.55 & 1.93 & 8.46 & 4.63 & 1.79 \\
\hline \hline$I G(5 \%)$ & 20.92 & 9.96 & 1.27 & 9.12 & 5.33 & 1.49 & 5.91 & 4.19 & 1.44 & 4.35 & 2.66 & 1.45 \\
$G P-I G(5 \%)$ & 23.08 & 11.75 & 1.73 & 8.93 & 5.42 & 1.51 & 6.16 & 4.06 & 1.56 & 5.09 & 3.50 & 1.55 \\
\hline \hline$I G(10 \%)$ & 21.80 & 11.29 & 1.23 & 8.96 & 5.00 & 1.44 & 5.86 & 3.85 & 1.42 & 4.32 & 2.81 & 1.46 \\
$G P-I G(10 \%)$ & 24.44 & 11.21 & 1.77 & 9.37 & 5.26 & 1.56 & 6.14 & 3.73 & 1.58 & 4.61 & 2.85 & 1.54 \\
\hline \hline$I G(20 \%)$ & 23.60 & 10.24 & 1.27 & 8.56 & 4.20 & 1.32 & 5.81 & 4.00 & 1.45 & 4.17 & 2.43 & 1.43 \\
$G P-I G(20 \%)$ & 25.65 & 10.97 & 1.73 & 9.44 & 4.68 & 1.68 & 6.00 & 4.17 & 1.55 & 4.53 & 2.73 & 1.57 \\
\hline \hline$I G(30 \%)$ & 24.50 & 10.95 & 1.24 & 9.21 & 5.89 & 1.38 & 5.98 & 4.35 & 1.48 & 4.16 & 2.27 & 1.34 \\
$G P-I G(30 \%)$ & 26.53 & 11.03 & 1.76 & 9.80 & 4.82 & 1.62 & 5.83 & 3.25 & 1.52 & 4.99 & 2.97 & 1.66 \\
\hline
\end{tabular}

(b) ARSD

\begin{tabular}{|c|ccc|ccc|ccc|ccc|}
\cline { 2 - 14 } \multicolumn{1}{c|}{} & \multicolumn{3}{c|}{$M=2$} & \multicolumn{3}{c|}{$M=3$} & \multicolumn{3}{c|}{$M=4$} & \multicolumn{3}{c|}{$M=5$} \\
\hline Procedure & Mean & StD & $\bar{R}$ & Mean & StD & $\bar{R}$ & Mean & StD & $\bar{R}$ & Mean & StD & $\bar{R}$ \\
\hline$I G(1 \%)$ & 19.25 & 9.81 & 1.07 & 12.65 & 6.80 & 1.00 & 10.37 & 4.97 & 1.02 & 9.20 & 4.49 & 1.03 \\
$G P-I G(1 \%)$ & 40.68 & 20.26 & 1.93 & 39.82 & 30.15 & 2.00 & 41.21 & 25.75 & 1.98 & 35.92 & 17.24 & 1.97 \\
\hline \hline$I G(5 \%)$ & 21.17 & 9.85 & 1.30 & 11.33 & 5.52 & 1.44 & 9.49 & 4.43 & 1.38 & 9.39 & 5.02 & 1.32 \\
$G P-I G(5 \%)$ & 23.36 & 11.81 & 1.70 & 12.62 & 10.68 & 1.56 & 12.22 & 7.68 & 1.62 & 12.77 & 7.80 & 1.68 \\
\hline \hline$I G(10 \%)$ & 22.06 & 11.20 & 1.24 & 11.21 & 4.98 & 1.46 & 10.02 & 4.65 & 1.39 & 9.39 & 4.25 & 1.46 \\
$G P-I G(10 \%)$ & 25.05 & 11.44 & 1.76 & 11.84 & 5.84 & 1.54 & 11.39 & 5.53 & 1.61 & 11.54 & 7.42 & 1.54 \\
\hline \hline$I G(20 \%)$ & 23.87 & 10.16 & 1.27 & 10.86 & 4.77 & 1.40 & 10.14 & 5.31 & 1.43 & 9.48 & 5.33 & 1.35 \\
$G P-I G(20 \%)$ & 26.38 & 11.20 & 1.73 & 12.20 & 5.11 & 1.60 & 11.74 & 8.26 & 1.57 & 12.32 & 7.32 & 1.65 \\
\hline$I G(30 \%)$ & 24.77 & 10.93 & 1.21 & 11.60 & 6.00 & 1.40 & 11.06 & 6.22 & 1.48 & 9.86 & 5.02 & 1.41 \\
$G P-I G(30 \%)$ & 27.45 & 11.58 & 1.79 & 12.72 & 5.47 & 1.60 & 11.44 & 6.67 & 1.52 & 12.43 & 7.49 & 1.59 \\
\hline \hline
\end{tabular}

Table 2: Average BRSD and ARSD and ranks $\bar{R}$ attained by IEMO/D coupled with $I G^{\text {th }}$ and $G D$ - $I G^{\text {th }}$ parameterized with different thresholds, when applied to WFG3 problem involving from 2 to 5 objectives.

(a) BRSD

\begin{tabular}{|c|ccc|ccc|ccc|ccc|}
\cline { 2 - 13 } \multicolumn{1}{c|}{} & \multicolumn{3}{c|}{$M=2$} & \multicolumn{3}{|c|}{$M=3$} & \multicolumn{3}{c|}{$M=4$} & \multicolumn{3}{c|}{$M=5$} \\
\hline Procedure & Mean & StD & $\bar{R}$ & Mean & StD & $\bar{R}$ & Mean & StD & $\bar{R}$ & Mean & StD & $\bar{R}$ \\
\hline$I G(1 \%)$ & 6.54 & 5.07 & 1.35 & 3.02 & 2.05 & 1.24 & 3.00 & 2.33 & 1.17 & 2.80 & 2.10 & 1.34 \\
$G P-I G(1 \%)$ & 8.87 & 6.68 & 1.65 & 4.65 & 3.18 & 1.76 & 4.50 & 3.40 & 1.83 & 3.45 & 2.59 & 1.66 \\
\hline \hline$I G(5 \%)$ & 4.81 & 3.60 & 1.61 & 2.40 & 1.93 & 1.59 & 2.20 & 1.79 & 1.49 & 2.40 & 1.92 & 1.45 \\
$G P-I G(5 \%)$ & 3.77 & 2.71 & 1.39 & 2.10 & 1.42 & 1.41 & 2.20 & 1.85 & 1.51 & 2.76 & 3.47 & 1.55 \\
\hline \hline$I G(10 \%)$ & 5.25 & 4.54 & 1.62 & 2.29 & 1.65 & 1.58 & 2.17 & 1.49 & 1.57 & 2.32 & 1.91 & 1.44 \\
$G P-I G(10 \%)$ & 4.09 & 3.16 & 1.38 & 2.04 & 1.47 & 1.42 & 2.17 & 1.75 & 1.43 & 2.42 & 2.37 & 1.56 \\
\hline \hline$I G(20 \%)$ & 4.15 & 3.14 & 1.61 & 2.38 & 1.89 & 1.50 & 2.30 & 1.62 & 1.51 & 2.22 & 1.82 & 1.39 \\
$G P-I G(20 \%)$ & 3.28 & 2.30 & 1.39 & 2.31 & 1.96 & 1.50 & 2.34 & 1.70 & 1.49 & 2.72 & 2.20 & 1.61 \\
\hline \hline$I G(30 \%)$ & 3.76 & 2.36 & 1.57 & 2.59 & 2.07 & 1.58 & 2.61 & 2.42 & 1.48 & 2.58 & 2.46 & 1.46 \\
$G P-I G(30 \%)$ & 3.66 & 2.91 & 1.43 & 2.31 & 1.73 & 1.42 & 2.43 & 1.83 & 1.52 & 2.87 & 2.45 & 1.54 \\
\hline \hline
\end{tabular}

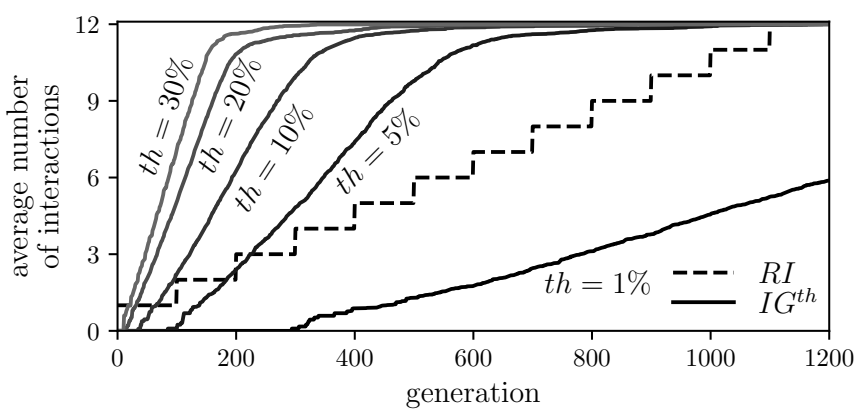

(a) IEMO/D with $R I$ and $I G^{t h}$

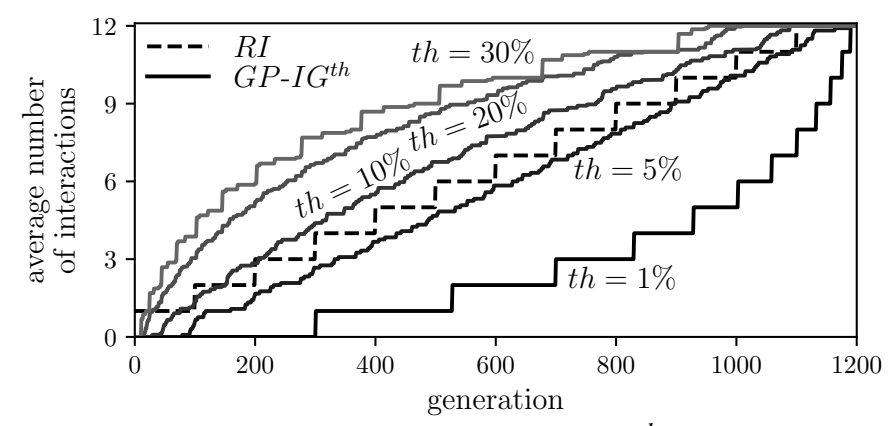

(b) IEMO/D with $R I$ and $G P-I G^{t h}$

Figure 1: Average number of interactions performed by IEMO/D coupled with (a) $I G^{t h}$ and (b) $G P-I G^{t h}$, along with $R I$, applied to WFG3 with $M=3$.

value system. Specifically, this function was used during the preference elicitation step to compare pairwise the reference solutions (b) ARSD

\begin{tabular}{|c|ccc|ccc|ccc|ccc|}
\cline { 2 - 15 } \multicolumn{1}{c|}{} & \multicolumn{4}{c|}{$M=2$} & \multicolumn{3}{c|}{$M=3$} & \multicolumn{3}{c|}{$M=4$} & \multicolumn{3}{c|}{$M=5$} \\
\hline Procedure & Mean & StD & $\bar{R}$ & Mean & StD & $\bar{R}$ & Mean & StD & $\bar{R}$ & Mean & StD & $\bar{R}$ \\
\hline$I G(1 \%)$ & 6.97 & 5.10 & 1.01 & 7.31 & 5.39 & 1.04 & 9.59 & 5.66 & 1.04 & 13.26 & 7.24 & 1.12 \\
$G P-I G(1 \%)$ & 40.72 & 37.89 & 1.99 & 29.16 & 20.68 & 1.96 & 29.77 & 15.19 & 1.96 & 26.40 & 15.03 & 1.88 \\
\hline \hline$I G(5 \%)$ & 5.23 & 3.93 & 1.62 & 7.21 & 5.64 & 1.50 & 9.76 & 5.70 & 1.44 & 13.67 & 7.26 & 1.44 \\
$G P-I G(5 \%)$ & 4.06 & 2.72 & 1.38 & 7.11 & 5.49 & 1.50 & 10.98 & 6.35 & 1.56 & 16.11 & 10.77 & 1.56 \\
\hline \hline$I G(10 \%)$ & 5.57 & 4.57 & 1.62 & 5.58 & 3.28 & 1.47 & 10.66 & 6.67 & 1.42 & 15.01 & 13.61 & 1.46 \\
$G P-I G(10 \%)$ & 4.47 & 3.39 & 1.38 & 6.52 & 4.32 & 1.53 & 12.37 & 9.85 & 1.58 & 15.46 & 9.82 & 1.54 \\
\hline \hline$I G(20 \%)$ & 4.47 & 3.20 & 1.55 & 6.37 & 4.53 & 1.48 & 10.65 & 6.37 & 1.53 & 14.71 & 9.74 & 1.37 \\
$G P-I G(20 \%)$ & 3.76 & 2.45 & 1.45 & 6.41 & 5.02 & 1.52 & 12.19 & 9.52 & 1.47 & 19.19 & 13.85 & 1.63 \\
\hline$I G(30 \%)$ & 4.20 & 2.62 & 1.58 & 6.76 & 4.56 & 1.57 & 12.91 & 10.84 & 1.47 & 14.63 & 7.49 & 1.37 \\
$G P-I G(30 \%)$ & 4.12 & 3.81 & 1.42 & 6.50 & 4.88 & 1.43 & 12.71 & 8.66 & 1.53 & 20.18 & 16.00 & 1.63 \\
\hline \hline
\end{tabular}

(i.e., a solution which attained a better score was considered as more preferred). To simulate the DM's preference intensity statements, apart from randomly pre-generating the objective weight vector $w^{D M}$, we drew two thresholds $t_{1}$ and $t_{2}\left(t_{1}<t_{2}\right)$ used to compare the differences between scores attained by the reference solutions. Specifically, when the difference was not greater than $t_{1}$, the preference was considered weak; when the difference was greater than $t_{1}$ but not greater than $t_{2}$, the preference was considered medium; if the difference exceeded $t_{2}$, the preference was considered strong. To ensure that these thresholds were well re-scaled according to the DM's preferences, we made them dependent on the scores assigned to utopia $u$ and nadir $n$ solutions for the underlying WFG problem. The scores were assigned as imposed by the $L_{\infty}^{w^{D M}}$-norm. Note that $u$ for any WFG problem is a zero vector, and thus the corresponding score is always 0 . Furthermore, we assumed that the thresholds should not be too large. In this way, even when the search conducted by IEMO/D is narrowed, and the differences between solutions are relatively small, the DM should be able to assign different preference intensities (i.e., weak, medium, or strong) to a pair of reference solutions. For the two above reasons, we drew the $t_{1}$ and $t_{2}$ thresholds from the $\left[L_{\infty}^{w^{D M}}(u)=0, L_{\infty}^{w^{D M}}(n)\right]$ interval and multiplied them by a reasonably small value of 0.1 . Thus generated thresholds were suitably re-scaled according to both the DM's value system and the underlying MOO problem.

\section{REFERENCES}

[1] M. Kadziński, M. K. Tomczyk, and R. Słowiński. 2020. Preference-based cone contraction algorithms for interactive evolutionary multiple objective optimization. Swarm and Evolutionary Computation 52 (2020), 100602.

[2] M. K. Tomczyk and M. Kadziński. 2020. Decomposition-based interactive evolutionary algorithm for multiple objective optimization. IEEE Transactions on Evolutionary Computation 24, 2 (2020), 320-334. 